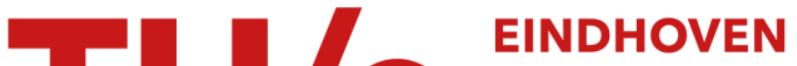 UNIVERSITY OF TECHNOLOGY
}

\section{Effect of film thickness on the far- and near-field optical response of nanoparticle-on-film systems}

\section{Citation for published version (APA):}

Armstrong, R. E., van Liempt, J. C., \& Zijlstra, P. (2019). Effect of film thickness on the far- and near-field optical response of nanoparticle-on-film systems. Journal of Physical Chemistry C, 123(42), 25801-25808.

https://doi.org/10.1021/acs.jpcc.9b06592

\section{Document license:}

CC BY-NC-ND

DOI:

10.1021/acs.jpcc.9b06592

Document status and date:

Published: 24/10/2019

\section{Document Version:}

Publisher's PDF, also known as Version of Record (includes final page, issue and volume numbers)

\section{Please check the document version of this publication:}

- A submitted manuscript is the version of the article upon submission and before peer-review. There can be important differences between the submitted version and the official published version of record. People interested in the research are advised to contact the author for the final version of the publication, or visit the $\mathrm{DOI}$ to the publisher's website.

- The final author version and the galley proof are versions of the publication after peer review.

- The final published version features the final layout of the paper including the volume, issue and page numbers.

Link to publication

\section{General rights}

Copyright and moral rights for the publications made accessible in the public portal are retained by the authors and/or other copyright owners and it is a condition of accessing publications that users recognise and abide by the legal requirements associated with these rights.

- Users may download and print one copy of any publication from the public portal for the purpose of private study or research.

- You may not further distribute the material or use it for any profit-making activity or commercial gain

- You may freely distribute the URL identifying the publication in the public portal.

If the publication is distributed under the terms of Article 25fa of the Dutch Copyright Act, indicated by the "Taverne" license above, please follow below link for the End User Agreement:

www.tue.nl/taverne

Take down policy

If you believe that this document breaches copyright please contact us at:

openaccess@tue.nl

providing details and we will investigate your claim. 


\title{
Effect of Film Thickness on the Far- and Near-Field Optical Response of Nanoparticle-on-Film Systems
}

\author{
Rachel E. Armstrong, * ${ }^{*}$ J. C. van Liempt, and Peter Zijlstra
}

Department of Applied Physics, Institute for Complex Molecular Systems, Eindhoven University of Technology, Postbus 513, 5600 MB Eindhoven, The Netherlands

\section{Supporting Information}

ABSTRACT: We study the near-field and far-field optical responses of nanoparticle-on-film systems using single-nanoparticle spectroscopy and numerical simulations. We find that the optical spectra contain three dominant modes: a transverse dipole, quadrupole mode, and a dominant vertical antenna mode. We vary the thickness of the metal film from 10 to 45 $\mathrm{nm}$ and find that the vertical antenna mode wavelength is nearly independent of the film thickness. In contrast, we find that the associated near-field enhancement in the gap between the particle and the film strongly depends on the film thickness. This trend is also observed in the far field where the

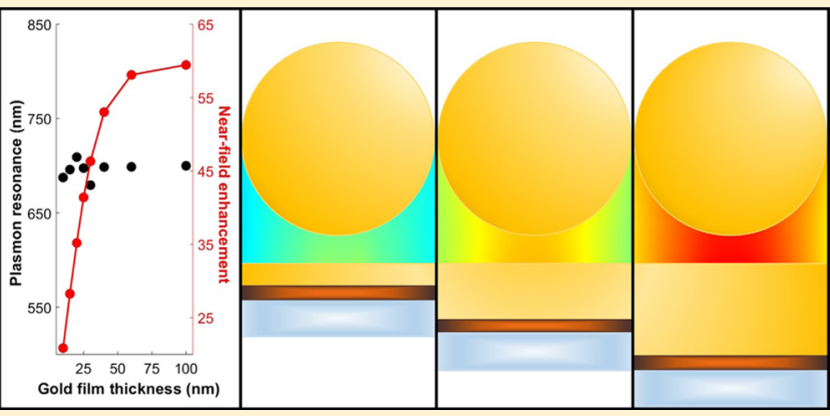
vertical antenna mode strongly increases in amplitude relative to the quadrupole for the increasing film thicknesses up to the skin depth of gold. These findings are in good agreement with a numerical model and pave the way to study field-mediated processes such as fluorescence, surface-enhanced Raman spectroscopy, and localized chemistry at the same resonance wavelength but at varying degrees of field enhancement.

\section{INTRODUCTION}

Applications ranging from biomedicine ${ }^{1-3}$ to electronics ${ }^{4}$ have capitalized on the generation of local electric fields by plasmonically coupled nanomaterials. The resonant excitation of neighboring plasmonic structures results in an enhanced field in the gap between the structures. ${ }^{1,5}$ The usefulness of these localized fields is exemplified by the signal enhancement of optical sensors, ${ }^{3,6-11}$ in the generation of exceptionally confined optical traps, ${ }^{12-14}$ surface-enhanced Raman spectroscopy (SERS) applications, ${ }^{15-18}$ and in target-specific biomedical imaging. ${ }^{19,20}$ To accomplish several of these applications, earlier studies have employed nanoparticle dimer systems and have shown experimentally ${ }^{1,21-24}$ and theoretically $^{11,25}$ that these dimers are sufficient for generating localized fields and with a large, red-shifted optical response. While there is no dispute on the usefulness of plasmonic dimer pairs, their construction is not simple. Complicated conjugation and low yield in the purification steps slow the progress of plasmonic nanoparticle dimers. ${ }^{21,23}$ On the other hand, multiple groups have investigated the physical mechanisms in generating these localized fields in simpler, nanoparticle-on-a-film (NPoF) systems, where the optical properties of noble-metal nanoparticles near a metal film are measured and modeled. ${ }^{4,5,26-38}$ From these studies, it is clear that the localization and field strength in the plasmonic gap are heavily dependent on the nanoparticle and film composition, ${ }^{4,26,27,32}$ the nanoparticle size and shape, ${ }^{15,28,39}$ the polarization/incident angle of excitation, ${ }^{4,15,20}$ and plasmonic gap distance. ${ }^{4,5,15,29-36,40}$ Studies that best quantify these dependencies are single-particle NPoF studies, in which spectral broadening due to ensemble averaging is removed and the spectral properties of the individual nanoparticles are compared to theory. $30,31,34,35,40$

Physical understanding of the plasmonic excitation is critical to its application. For instance, it is observed experimentally and predicted theoretically that the nanoparticle's localized surface plasmon resonance (LSPR) red-shifts with a decreased plasmonic gap above a metal film due to coupling to the particle's vertical antenna mode. ${ }^{4,15,26,27,29-37}$ Quantification of the gap-dependent red shift is necessary for the integration of NPoF systems into wavelength-specific applications like biomedical imaging, where the scattering wavelength of the nanoparticles must lie in the biological tissue window. ${ }^{2}$

Here, we show the modulation of the optical properties of the NPoF systems by tuning the gold film thickness. Hyperspectral microscopy was used to collect single-particle scattering spectra of $100 \mathrm{~nm}$ gold nanoparticles on gold films with varying thicknesses. The use of single-particle hyperspectral microscopy allows for the spectroscopy of hundreds of nanoparticles simultaneously, providing a statistical means to investigate particle-to-particle heterogeneity. We find that the amplitude of the nanoparticle's vertical antenna mode relative to the quadrupole increases for film thicknesses below the skin depth of gold and remains constant for thicker films.

Received: July 11, 2019

Revised: September 24, 2019

Published: September 24, 2019 

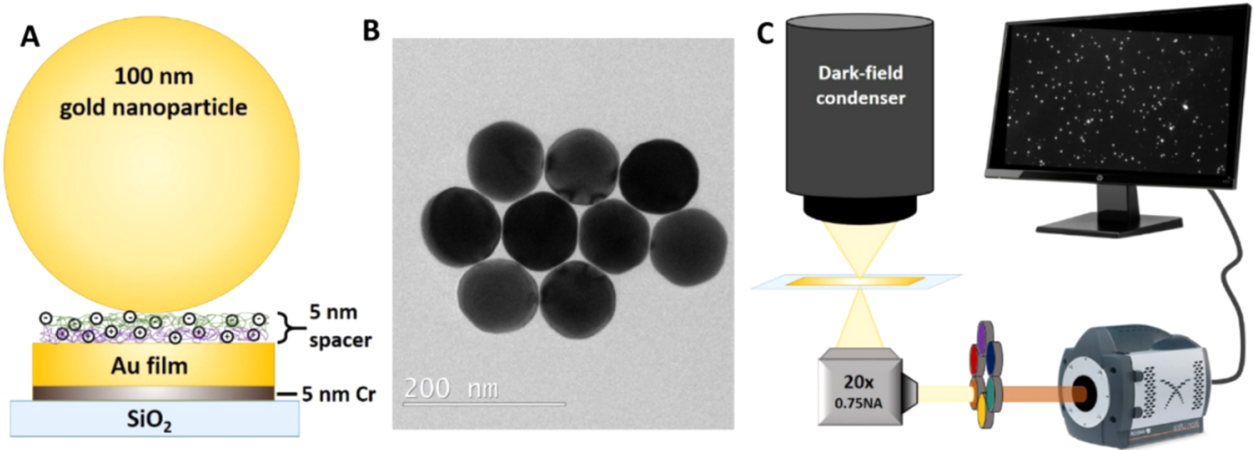

Figure 1. (A) NPoF configuration. A thin gold film is evaporated onto a $5 \mathrm{~nm}$ chromium layer on a glass coverslip. Alternating PDADMAC (purple, positively charged) and PSS (green, negatively charged) polymer layers are assembled on top of the gold film. One hundred nanometer diameter gold nanoparticles are spin-coated onto the polymer spacer layer. (B) Transmission electron microscopy image of ultrauniform $100 \mathrm{~nm}$ gold particles. (C) Sketch of the dark-field single-particle hyperspectral microscopy setup.

Interestingly, the resonance wavelength is nearly independent of film thickness, which is confirmed by numerical simulations. Simulated near-field spectra indicate that modulating the film thickness provides the opportunity to systematically vary the near-field enhancement without affecting the resonance wavelength. This provides a convenient way to investigate how the magnitude of the field enhancement affects the fluorescence and SERS signals, without the need to modify chemical protocols to modulate the gap spacing or particle size.

\section{EXPERIMENTAL METHODS}

2.1. Materials. All materials were purchased from SigmaAldrich unless otherwise noted. Ultra Uniform, PEG carboxyl $(100 \mathrm{~nm})$ gold spheres were purchased from nanoComposix.

2.2. Gold Film Preparation and Nanoparticle Deposition. Dark-field hyperspectral microscopy of individual 100 $\mathrm{nm}$ nanoparticles was performed on gold films of various thicknesses (Figure 1). Thin gold films (10, 15, 30, and $45 \mathrm{~nm}$ ) were prepared via thermal evaporation with chromium $(5 \mathrm{~nm}$ thick) as an adhesion layer. The film thickness was measured using a Dektak XT profilometer by blocking gold deposition of a small region with tape and measuring across the edge when the tape was removed (SF1). Additional characterization by UV-vis spectroscopy is shown in the Supporting Information (SF2). We deposited a thin polymeric spacer on top of the film, consisting of four polymer layers of, alternating, positively charged poly(diallyldimethylammonium chloride) (PDADMAC) layer followed by negatively charged poly(sodium 4styrenesulfonate) (PSS) layer. The polymer layer was characterized via ellipsometry to have a final thickness of 5 $\mathrm{nm}$ (SF3). The gold nanoparticles' dense ligand coating (HSPEG12-COOH) likely adds minimally to the gap spacing. After rinsing the deposited polymer film using $1 \mathrm{M} \mathrm{NaCl}$ and methanol, $100 \mathrm{~nm}$ ultrauniform nanoparticles (Nanocomposix, Figure 1B) are deposited via spin-coating. Atomic force microscopy (AFM) was performed after spin-coating to confirm the presence of single, nonclustered nanoparticles (SF4).

2.3. Single-Particle Hyperspectral Microscopy. Samples were placed in an inverted optical microscope, and the dry sample was illuminated with white light through a dark-field condenser (NA $=0.8-0.95$ ) (Figure 1C). The scattering intensity from $>100$ nanoparticles per sample was collected with a $20 \times($ NA 0.75$)$ objective and imaged on an EMCCD camera. To measure the wavelength-dependent scattered intensity, bandpass filters were placed in front of the EMCCD camera at $10 \mathrm{~nm}$ intervals ranging from 520 to 860 $\mathrm{nm}$ (Figure 1C). Individual nanoparticle spectra were generated by plotting the sum of the scattered intensity for the nanoparticle's region of interest at each bandpass filter wavelength.

2.4. Boundary Element Method (BEM) Simulations. Numerical simulations of the optical properties of gold nanoparticles on gold films were performed using the boundary element method (BEM) in MATLAB using the MNPBEM toolbox. ${ }^{41,42}$ The MNPBEM toolbox solves Maxwell's equations but simplifies the system by assuming only the interfaces between objects. ${ }^{41,42}$ The simulated geometry consisted of a glass substrate, on top of which a 5 $\mathrm{nm}$ layer of chromium, a gold film, and a $5 \mathrm{~nm}$ polymer spacer were modeled as flat layers with a $100 \mathrm{~nm}$ gold sphere on top (SF5). The dielectric constants of the glass substrate and polymer spacer layer were defined as $n=1.52$ and 1.5, respectively. The spectrum was recorded with an angle of illumination of $70^{\circ}$ with respect to the normal of the substrate. While the dark-field condenser actually illuminates with a mixture of incident angles $\left(53-72^{\circ}\right)$ determined by the numerical aperture of the condenser $(\mathrm{NA}=0.8-0.95)$, best agreement with experimental results is observed for angles around $70^{\circ}$, indicating that the setup does not produce a cone of light with equal intensity across the full angular range. An analysis of the effect of the illumination angle on the far-field spectrum calculations is described in more detail in the Supporting Information (SF5). The spectra were calculated for unpolarized illumination by assuming a linear superposition of s- and p-polarized light.

\section{RESULTS AND DISCUSSION}

3.1. Plasmon Mode Identification. In Figure 2, we show a typical scattering spectrum obtained for a single particle on a $45 \mathrm{~nm}$ gold film and a $5 \mathrm{~nm}$ chromium film, with a polymeric spacer of $5 \mathrm{~nm}$. The spectra typically display two characteristic modes at around 550 and $700 \mathrm{~nm}$, with a weak shoulder visible between 550 and $600 \mathrm{~nm}$, as shown in Figure 2. The simulated spectra predict three spectrally distinct plasmonic modes: a quadrupole, a transverse dipole, and a vertical antenna mode. Surface charge distribution calculations at the wavelengths indicated in the spectrum are shown in Figure 2 (right) and clearly identify the quadrupole mode at $530 \mathrm{~nm}$, the transverse dipole at $580 \mathrm{~nm}$, and the vertical antenna mode at $700 \mathrm{~nm}$. 


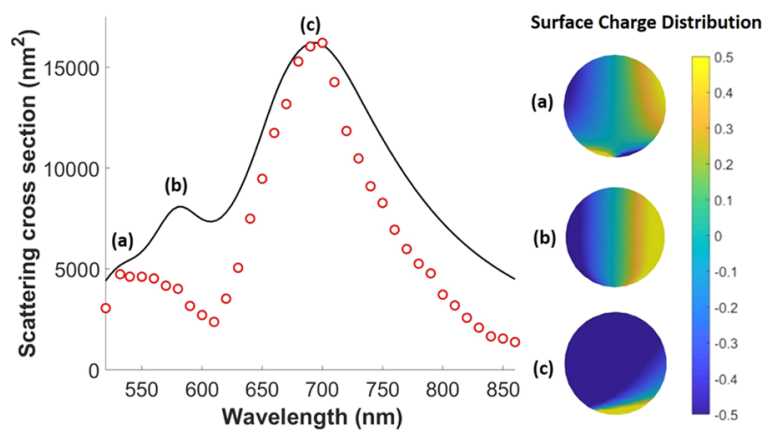

Figure 2. (Left) Typical measured scattering spectrum (red circles) for a $100 \mathrm{~nm}$ particle on a $45 \mathrm{~nm}$ thick film, with a spacer of $5 \mathrm{~nm}$. The solid line shows the calculated spectrum (boundary element method). The calculated scattering spectra are normalized to the experimental spectrum. (Right) Surface charge density on the surface of the sphere at the three indicated wavelengths. The sphere projections were calculated with the BEM geometry described in Section 2.4; the gold film beneath the nanoparticle is not shown.

Although broadened (see Section 3.3.2), the center wavelength of the strong vertical antenna mode is in good agreement with the experimentally measured spectrum. Both the vertical antenna mode and the quadrupole mode are not observed in single-particle spectra in the absence of a gold film (SF6). The vertical antenna mode is indeed induced by coupling between the particle and the film, whereas the (usually dark) quadrupole mode radiates more efficiently due to symmetry breaking induced by the presence of the metal film. ${ }^{28,43}$ Interestingly, we consistently observe only a weak contribution from the transverse dipolar mode, the origins of which are unclear. Other single-particle NPoF studies have reported this phenomenon also; however, the transverse resonance is often identified as the mode at $540 \mathrm{~nm}$, negating the contribution of the quadrupole mode. ${ }^{28,30}$ Surface charge distribution calculations clearly identify the quadrupole mode (Figure $2(\mathrm{a}))$ at $530 \mathrm{~nm}$ here. Therefore, we believe the quadrupole and transverse modes are spectrally overlapped at $530 \mathrm{~nm}$, but simulations do not effectively predict the transverse resonance wavelength.

From the BEM calculations, we also observe a clear effect of introducing a $5 \mathrm{~nm}$ chromium layer into the BEM calculations. Calculated far-field spectra of all films with and without the chromium layer are presented in the Supporting Information (SF7). Simulated spectra of the 10 and $15 \mathrm{~nm}$ gold films are dominated by the lossy chromium layer, whereas the 30 and 45 $\mathrm{nm}$ gold film spectra are comparable with and without chromium. This is unsurprising, as the 30 and $45 \mathrm{~nm}$ films are nearer to the skin depth of gold $(42 \mathrm{~nm})$ and the nanoparticle's mirrored dipole into the film does not interact strongly with the chromium layer.

3.2. Particle-to-Particle Heterogeneity. The use of single-particle hyperspectral microscopy presents the opportunity to study large numbers of particles and isolate sources of heterogeneity observed in previous studies. We show the single-particle spectra of 117 particles in Figure 3, acquired on a $45 \mathrm{~nm}$ thick Au film with a polymeric spacer of $5 \mathrm{~nm}$. We observe a reproducible optical response with a typical coefficient of variation of $50 \%$ in scattered intensity and $\leq 3 \%$ in plasmon energy of the vertical cavity mode (mean $\pm \mathrm{sd}$ of $683 \pm 15 \mathrm{~nm}$, or $1.83 \pm 0.04 \mathrm{eV}$ ). This variability was similar for other samples with different gold film thicknesses as studied later, and we will now first analyze the possible origins of it.

Other groups have suggested that nanoparticle size distribution, $^{28,44}$ presence of facets, ${ }^{28,39,44}$ variations in the nanoparticle-film gap distance, ${ }^{27,29-32,34-36}$ and Au film roughness ${ }^{45}$ are likely contributors to the particle-to-particle variability observed in single NPoF systems. The variability in the vertical antenna mode that we find is however less than the variability reported in the literature. ${ }^{30} \mathrm{We}$ attribute this to the usage of ultrauniform particles (Nanocomposix) that are nearly perfectly spherical, and their diameter exhibits a coefficient of variation of only $\sim 5 \%$.

Earlier studies have reported high variability in singlenanoparticle spectra when resting near protrusions in the gold film surface. ${ }^{45}$ To rule out this contribution, we investigated the roughness of the metal film by AFM prior to polymer deposition. Full AFM details on the surface roughness assessment are described in the Supporting Information (SF8, Table S1). An earlier study by Ivanova et al. demonstrates that vertical surface roughness in metal films is best assessed by two AFM measurements, average roughness $\left(R_{\mathrm{a}}\right)$ and root-mean-square roughness $\left(R_{\mathrm{q}}\right) \cdot{ }^{47}$ For all gold film thicknesses assessed by AFM, exceptionally low $R_{\mathrm{a}}(0.3-1 \mathrm{~nm})$ and $R_{\mathrm{q}}(0.2-0.9 \mathrm{~nm})$ indicate that the gold films are nearly atomically smooth, ruling out a strong contribution to the observed particle-to-particle variation.

We therefore attribute the residual heterogeneity to small differences in the nanoparticle-film gap size, ${ }^{27,29,30,34-36,45}$ in
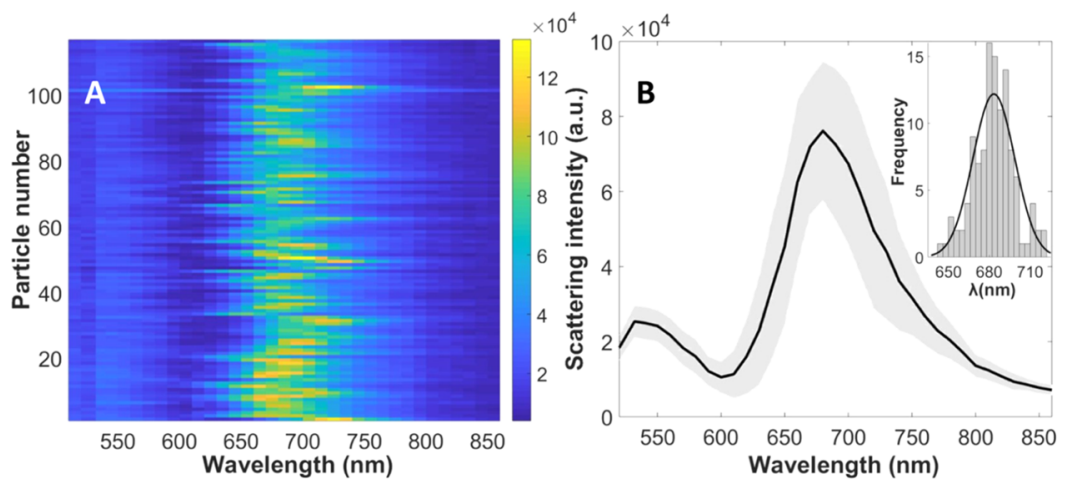

Figure 3. (A) Waterfall plot of single-particle scattering spectra for 117 particles on a $45 \mathrm{~nm}$ thick film with a $4 \mathrm{~nm}$ polymeric spacer. (B) Mean (black line) and standard deviation (shaded area) of the spectra. The inset shows the measured distribution of the center wavelength of the vertical antenna mode $($ mean $\pm \mathrm{sd}=683 \pm 15 \mathrm{~nm})$. 

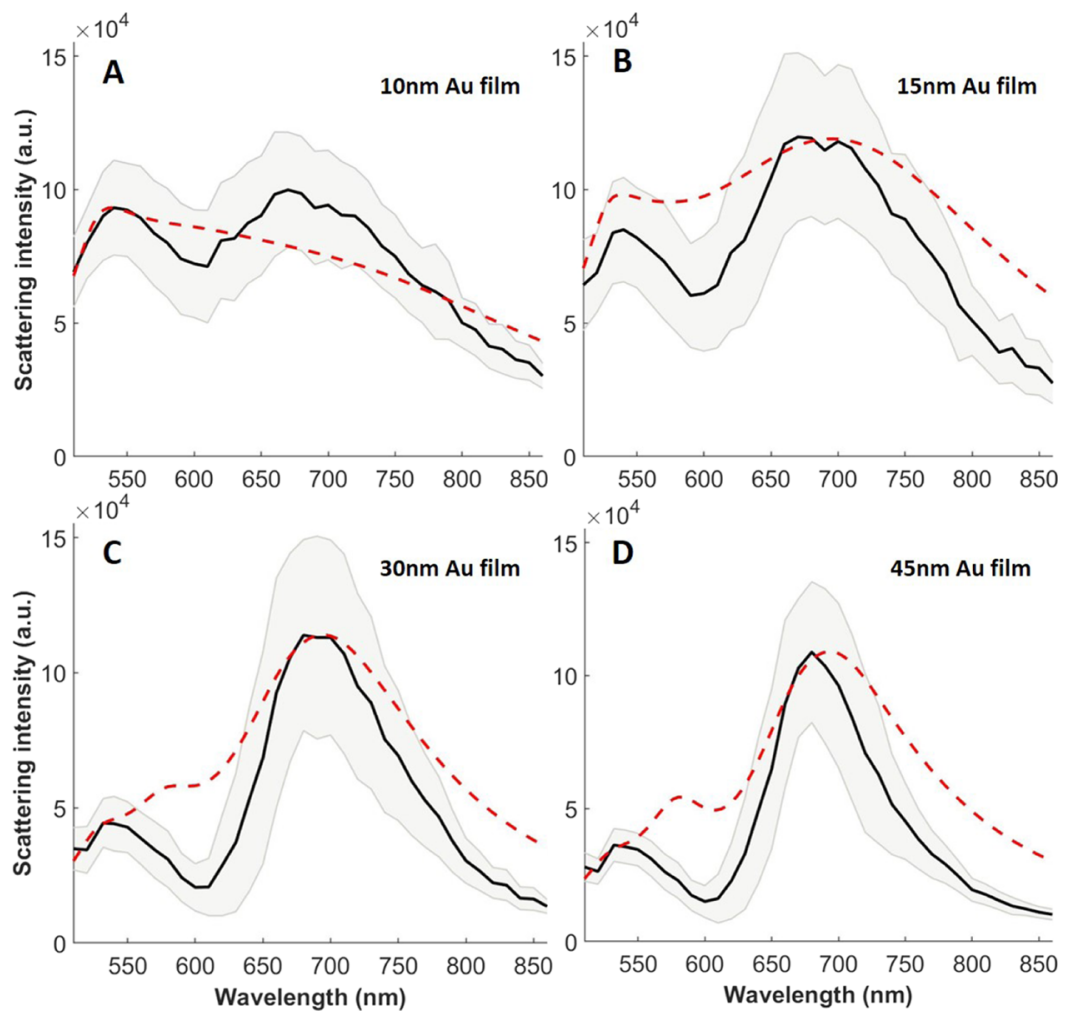

Figure 4. (A-D) Average single-nanoparticle spectra (black lines) and the standard deviation of the average at each point (shaded area). Red dotted lines show the predicted far-field spectrum calculated using BEM simulations. In (A), 115 nanoparticles on a $10 \mathrm{~nm}$ gold film; in (B), 116 nanoparticles on a $15 \mathrm{~nm}$ film; in (C), 136 particles on a $30 \mathrm{~nm}$ film; and in (D), 117 particles on a $45 \mathrm{~nm}$ film were measured.
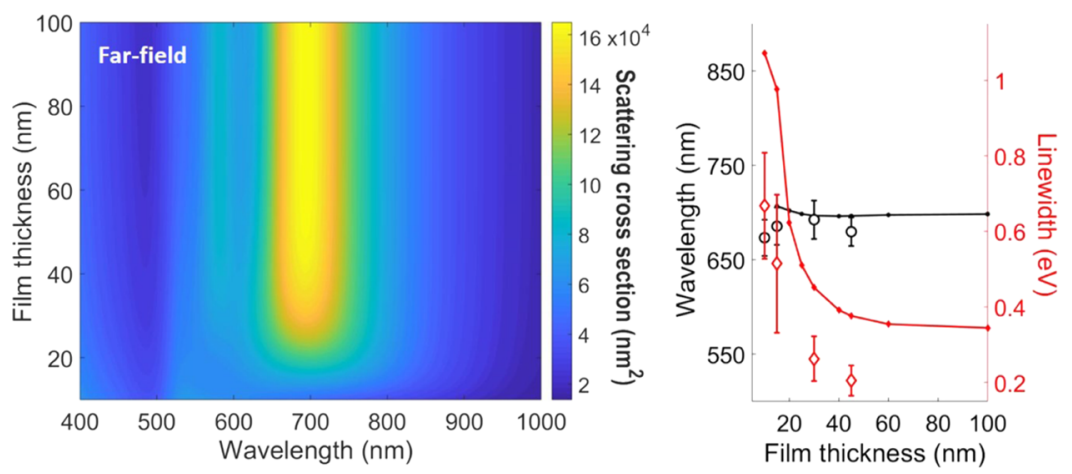

Figure 5. (Left) Calculated far-field spectra of a gold nanoparticle with a $5 \mathrm{~nm}$ spacer above gold films of varying thicknesses. (Right) BEMcalculated vertical mode resonance (black line) and line width (red line) as a function of gold film thickness. Values were extracted from the calculated far-field spectra by fitting to a three-Lorentzian curve. Experimental values for the wavelength are denoted by circles (black), and the experimentally measured line widths are denoted by red diamonds (red). Error bars on the experimental data are the standard deviations of the normal distribution for all single-particle spectra measured in the sample. Note: A BEM-calculated value for the vertical resonance on a $10 \mathrm{~nm}$ film is not given, as a clear resonance mode is not observed in the far-field spectra and peak values could not be extracted.

combination with the slight asphericity of a fraction of the particles (see Figure 1B). In our studies where a polymer spacer is incorporated, inhomogeneity in the polymer layer across the sample could contribute to spacer differences of a few nanometers, which has been reported with this polymer previously. ${ }^{46}$ In fact, a mere $1 \mathrm{~nm}$ decrease in the gap could result in $\sim 25 \mathrm{~nm}$ shifts in the vertical antenna mode on thicker $(<30 \mathrm{~nm})$ gold films. ${ }^{5,26,27,29-31}$ Future measurements to further suppress the heterogeneity arising from these sources would require an atomic-level control over the nanoparticle shape, orientation, surface ligand structure, and spacing. The latter could be achieved using, e.g., atomic layer deposition wherein atomically flat spacers can be deposited across large areas. ${ }^{48}$

3.3. Effect of Gold Film Thickness. 3.3.1. Plasmon Wavelength. Hyperspectral microscopy was then employed to monitor the changes in single-particle spectra as a function of film thickness. Scattering spectra of at least 100 nanoparticles on each gold film thickness were measured and plotted in Figure 4, where we show the mean and standard deviation of all nanoparticles in a field of view of the microscope. Average nanoparticle spectra on all film thicknesses display two identifiable peaks at $\sim 540$ and $\sim 690 \mathrm{~nm}$. The peak identities in each spectra based on BEM calculations are the quadrupole (and spectrally convoluted the transverse dipolar plasmon) at 
$\sim 540 \mathrm{~nm}$ and the vertical antenna mode at $\sim 690 \mathrm{~nm}$. BEM predictions for this NPoF system are also shown in Figure 4 (red dotted lines), where calculated plasmon wavelengths correspond well with measurements.

While previous reports show a strong sensitivity of the vertical antenna mode wavelength to gap distance, the vertical antenna mode wavelength is interestingly independent of film thickness (Figure 5). More detailed BEM calculations of this $\mathrm{NPoF}$ configuration (see Figure 5) predict that the vertical antenna mode wavelength is independent of the film thickness for a large range of film thicknesses, as we observe experimentally. Additionally, there is a clear increase in the quadrupole mode magnitude with increasing film thickness. The ratio of scattering intensity between the quadrupole and vertical antenna mode is approximately $1: 1$ for the $10 \mathrm{~nm}$ gold film and increases to approximately $1: 3$ for the $45 \mathrm{~nm}$ film. The calculated spectra (Figure 4, red dotted lines) and the hyperspectral measurements (black lines) show a good correlation in this regard, as BEM calculations predict nearly identical ratios (0.85:1 and 1:3.0, respectively).

3.3.2. Plasmon Linewidth. Surprisingly, we consistently measure a smaller linewidth $(\Gamma)$ for the vertical antenna mode than predicted by the simulations (Figures 2 and 5). Sobhani et al. describe, albeit for aluminum nanoparticles, that hybridization of the quadrupole and dipole modes leads to linewidth narrowing and that this phenomena is most clear when tracking the LSPR linewidth as a function of nanoparticle-film spacing. ${ }^{49}$ We find that the calculated vertical mode linewidth for gold nanoparticles does not change substantially when the vertical antenna mode shifts away from the quadrupole with decreasing gap spacing (SF9). Therefore, we do not believe the experimentally narrow linewidth is caused by a physical mechanism, but rather current models overpredict the linewidth.

Other groups have observed this same discrepancy using other numerical methods, with some reporting simulated linewidths to be over $\sim 2 \times$ larger than their single-particle NPoF measurements $(175 \mathrm{meV}$ observed vs $400 \mathrm{meV}$ calculated). ${ }^{28,30,34,35,43,50}$ Of influence may be the optical functions of gold used in these numerical simulations, which vary in the nanodomain and by the deposition method. ${ }^{51-53}$ Indeed, applying functions from the work of Palik, rather than Johnson and Christy, produces a far-field spectrum with a significantly narrower vertical antenna mode $\left(\Gamma_{\mathrm{J \& C}}=340 \mathrm{meV}\right.$; $\left.\Gamma_{\text {Palik }}=272 \mathrm{meV}\right)(\mathrm{SF} 10)$. The differences between the Palik and Johnson and Christy constants are small and are isolated to the regime where interband transitions dominate $(2.5-4.5$ eV) (SF10). This provides a hint that changes in the interband transitions, or perhaps modifications of radiative damping may be the dominant mechanisms causing the often observed discrepancy.

3.3.3. Near-Field Response. The use of NPoF systems to study the coupling between localized modes and emitters in the gap has received considerable attention in the past years. ${ }^{15}$ The main advantage of NPoF systems is their ease of assembly and the considerable field enhancements that can be generated in the nanometer gap between the particle and the film. ${ }^{15}$ NPoF systems have therefore been used to study the coupling between localized modes and emitters for fluorescence enhancement, ${ }^{54-57}$ SERS, ${ }^{15,16}$ and have even resulted in the demonstration of single-molecule room-temperature strong coupling. ${ }^{56}$
Such a coupling between a localized resonance and an emitter is governed by the near-field rather than the far-field spectra. ${ }^{58}$ We therefore simulated the near-field optical response of NPoF systems for varying film thicknesses. The results are shown in Figure 6 where we plot the near-field

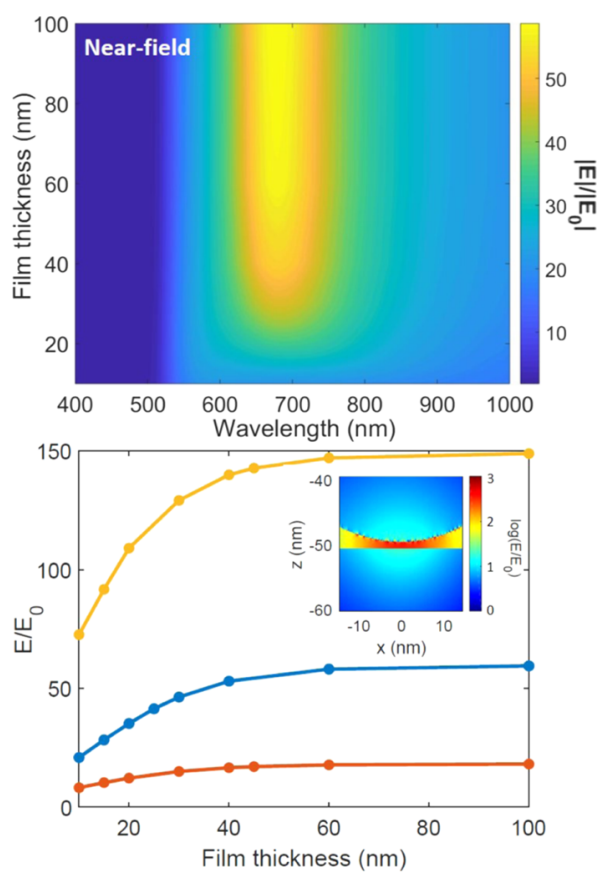

Figure 6. (Top) Calculated near-field spectra of a gold nanoparticle with a $5 \mathrm{~nm}$ spacer above gold films of varying thicknesses. (Bottom) Relationship between the near-field enhancement on resonance with the vertical antenna mode and film thickness for three particle-film spacings: $1 \mathrm{~nm}$ (yellow), $5 \mathrm{~nm}$ (blue), and $10 \mathrm{~nm}$ (red). The field strength is calculated at the centermost point in the gap. The inset shows an example of the near-field calculation in the nanoparticlefilm gap (gold film thickness is $45 \mathrm{~nm}$ with $1 \mathrm{~nm}$ gap spacing).

spectra and wavelength-dependent near-field enhancement in the center of the gap. For the near-field spectra, we observe a trend similar to that for the far-field spectra: for increasing film thickness, the near-field enhancement increases up to the skin depth of gold, after which both the spectral profile and the absolute enhancement approach an asymptote. As expected, the near-field spectrum is substantially broadened compared to the far-field spectrum due to constructive interference between the incoming light field and the near field on the red wing of the vertical antenna mode. Interestingly, we observe the nearfield enhancements up to $\sim 30 \times$ in the gap for the transverse dipolar mode at $\sim 580 \mathrm{~nm}$, substantially higher than the maximum field enhancement of $5 \times$ on the surface of an isolated gold sphere.

The strongest fields are logically observed for the vertical antenna mode that exhibits a resonance wavelength away from the interband transitions in gold and thus suffers from less losses than the transverse dipole and quadrupole modes. We therefore plot the field enhancement in the gap on resonance with the vertical cavity mode; see Figure 6 . As a function of film thickness, we observe a gradual increase in the field enhancement, as expected from the increased dipole moment already observed in the far-field spectra. The near-field enhancement gradually increases from $\frac{|E|}{\left|E_{0}\right|}=20$ for a $10 \mathrm{~nm}$ 
gold film until it saturates at $\frac{|E|}{\left|E_{0}\right|}=60$ for a thicker $45 \mathrm{~nm}$ film. Plotting the maximum near-field enhancement as a function of film thickness for multiple gap spacings (Figure 6) confirms that the limiting factor in the near-field enhancement is the penetration depth of gold $(42 \mathrm{~nm})$. For all simulated gap spacings, the maximum near-field enhancement saturates at a gold film thickness of $\sim 45 \mathrm{~nm}$. We additionally simulate the vertical antenna mode resonance as a function of gap spacing across all film thicknesses in the Supporting Information (SF11). The vertical antenna mode is largely independent of film thickness (as observed for the $5 \mathrm{~nm}$ gap spacing) but redshifts to $>800 \mathrm{~nm}$ for small gap sizes $(<2 \mathrm{~nm})$.

The fact that the field enhancement can be tuned while keeping the resonance wavelength constant provides the opportunity to straightforwardly study the effect of nanophotonic environments on fluorescence and SERS as a function of field enhancement. There is no need to change the gap size, material, or particle immobilization chemistry, which often affect the resonance wavelengths of the NPoF system and change the spectral overlap between the emitter and the resonance. Such studies will be advantageous to unravel, e.g., the transition from the weak coupling to the strong coupling regime ${ }^{56}$ and to study plasmon-enhanced electrochemistry ${ }^{59,60}$ and optical metasurfaces ${ }^{61}$ with varying degrees of field enhancement. In addition, there is no need to use films thicker than $\sim 45 \mathrm{~nm}$ (especially where transmission through the film is desirable), as gold's skin depth at $\sim 40 \mathrm{~nm}$ is the limiting property for maximum electric field generation at all NPoF spacings.

\section{CONCLUSIONS}

We have investigated the effect of gold film thickness on the near-field and far-field spectra of nanoparticle-on-film systems. By employing hyperspectral microscopy, we obtained the spectra of hundreds of particles, allowing us to establish that the heterogeneity in the optical response is strongly suppressed compared to the literature reports due to the use of ultrauniform gold particles. We attribute the residual heterogeneity to the slight remaining asphericity of a fraction of the particles, in combination with variations in the gap spacing induced by a spatially varying thickness of the polymer spacer layer. Our results are in agreement with other reports that find a substantially narrower line width than predicted by simulations. The origin of this remains unclear, but we hypothesize that it is caused by an inaccurate dielectric function of gold for thin films. On the other hand, BEM calculations predict the vertical mode resonance remarkably well. Over a range of film thicknesses we find, experimentally and via simulations, that the resonance wavelength of the vertical antenna mode is nearly independent of the film thickness, whereas its dipole moment and associated near-field enhancement strongly depend on it. Further analysis of the near-field dependence on film thickness indicates saturation of the near-field enhancement for $45 \mathrm{~nm}$ films, regardless of gap spacing. Overall, our results provide an improved understanding of the rich physics underlying NPoF systems and pave the way to study field-mediated processes such as fluorescence, SERS, and localized chemistry at the same resonance wavelength but at varying degrees of field enhancement.

\section{ASSOCIATED CONTENT}

\section{Supporting Information}

The Supporting Information is available free of charge on the ACS Publications website at DOI: 10.1021/acs.jpcc.9b06592.

Gold film characterization; UV-vis and AFM roughness analysis; polymer characterizations; single-nanoparticle characterization; BEM simulations; and refractive indices of gold (PDF)

\section{AUTHOR INFORMATION}

\section{Corresponding Author}

*E-mail: r.e.armstrong@tue.nl.

ORCID

Rachel E. Armstrong: 0000-0002-2356-8753

Peter Zijlstra: 0000-0001-9804-2265

Notes

The authors declare no competing financial interest.

\section{ACKNOWLEDGMENTS}

R.E.A., J.C.v.L., and P.Z. acknowledge financial support from The Netherlands Organization for Scientific Research (NWO VIDI). The authors thank Michael Beuwer and Claudia Schot for providing the AFM images for this study. We also thank Marcel Verheijen for TEM imaging, and Solliance and the Dutch province of Noord Brabant are acknowledged for funding the TEM facility.

\section{REFERENCES}

(1) Maurer, T.; Adam, P. M.; Leveque, G. Coupling between plasmonic films and nanostructures: from basics to applications. Nanophotonics 2015, 4, 361-382.

(2) Spackova, B.; Wrobel, P.; Bockova, M.; Homola, J. Optical Biosensors Based on Plasmonic Nanostructures: A Review. Proc. IEEE 2016, 104, 2380-2408.

(3) Guo, L. H.; Jackman, J. A.; Yang, H. H.; Chen, P.; Cho, N. J.; Kim, D. H. Strategies for enhancing the sensitivity of plasmonic nanosensors. Nano Today 2015, 10, 213-239.

(4) Wang, T.; Nijhuis, C. A. Molecular electronic plasmonics. Appl. Mater. Today 2016, 3, 73-86.

(5) Taylor, A. D.; Lu, C.; Geyer, S.; Carroll, D. L. Thin film based plasmon nanorulers. Appl. Phys. Lett. 2016, 109, No. 013105.

(6) Anker, J. N.; Hall, W. P.; Lyandres, O.; Shah, N. C.; Zhao, J.; Van Duyne, R. P. Biosensing with plasmonic nanosensors. Nat. Mater. 2008, 7, 442-453.

(7) Hill, R. T. Plasmonic biosensors. Wiley Interdiscip. Rev.: Nanomed. Nanobiotechnol. 2015, 7, 152-168.

(8) Taylor, A. B.; Zijlstra, P. Single-Molecule Plasmon Sensing: Current Status and Future Prospects. ACS Sens. 2017, 2, 1103-1122.

(9) Cao, Y.; McDermott, M. T. Femtomolar and selective dopamine detection by a gold nanoparticle enhanced surface plasmon resonance aptasensor. bioRxiv 2018, DOI: 10.1101/273078.

(10) Chen, Z.; Peng, Y.; Cao, Y.; Wang, H.; Zhang, J.-R.; Chen, H.Y.; Zhu, J.-J. Light-Driven Nano-oscillators for Label-Free SingleMolecule Monitoring of MicroRNA. Nano Lett. 2018, 18, 37593765.

(11) Zijlstra, P.; Orrit, M. Single metal nanoparticles: optical detection, spectroscopy and applications. Rep. Prog. Phys. 2011, 74, No. 106401.

(12) Verschueren, D. V.; Pud, S.; Shi, X.; De Angelis, L.; Kuipers, L.; Dekker, C. Label-Free Optical Detection of DNA Translocations through Plasmonic Nanopores. ACS Nano 2019, 13, 61-70.

(13) Shi, X.; Verschueren, D. V.; Dekker, C. Active Delivery of Single DNA Molecules into a Plasmonic Nanopore for Label-Free Optical Sensing. Nano Lett. 2018, 18, 8003-8010. 
(14) Ivinskaya, A.; Petrov, M. I.; Bogdanov, A. A.; Shishkin, I.; Ginzburg, P.; Shalin, A. S. Plasmon-assisted optical trapping and antitrapping. Light: Sci. Appl. 2017, 6, No. e16258.

(15) Baumberg, J. J.; Aizpurua, J.; Mikkelsen, M. H.; Smith, D. R. Extreme nanophotonics from ultrathin metallic gaps. Nat. Mater. 2019, 18, 668-678.

(16) Jones, S. T.; Taylor, R. W.; Esteban, R.; Abo-Hamed, E. K.; Bomans, P. H. H.; Sommerdijk, N. A. J. M.; Aizpurua, J.; Baumberg, J. J.; Scherman, O. A. Gold Nanorods with Sub-Nanometer Separation using Cucurbit n uril for SERS Applications. Small 2014, 10, 42984303.

(17) Li, L.; Hutter, T.; Steiner, U.; Mahajan, S. Single molecule SERS and detection of biomolecules with a single gold nanoparticle on a mirror junction. Analyst 2013, 138, 4574-4578.

(18) Di Martino, G.; Turek, V. A.; Tserkezis, C.; Lombardi, A.; Kuhn, A.; Baumberg, J. J. Plasmonic response and SERS modulation in electrochemical applied potentials. Faraday Discuss. 2017, 205, 537-545.

(19) Aaron, J.; Nitin, N.; Travis, K.; Kumar, S.; Collier, T.; Park, S. Y.; Jose-Yacaman, M.; Coghlan, L.; Follen, M.; Richards-Kortum, R.; Sokolov, K. Plasmon resonance coupling of metal nanoparticles for molecular imaging of carcinogenesis in vivo. J. Biomed. Opt. 2007, 12, No. 034007.

(20) Kumar, A.; Kim, S.; Nam, J.-M. Plasmonically Engineered Nanoprobes for Biomedical Applications. J. Am. Chem. Soc. 2016, 138, 14509-14525.

(21) Lee, S. E.; Chen, Q.; Bhat, R.; Petkiewicz, S.; Smith, J. M.; Ferry, V. E.; Correia, A. L.; Alivisatos, A. P.; Bissell, M. J. Reversible Aptamer-Au Plasmon Rulers for Secreted Single Molecules. Nano Lett. 2015, 15, 4564-4570.

(22) Zhang, S.; Reinhard, B. M. Characterizing Large-Scale Receptor Clustering on the Single Cell Level: A Comparative Plasmon Coupling and Fluorescence Superresolution Microscopy Study. J. Phys. Chem. B 2019, 123, 5494-5505.

(23) Ye, W. X.; Gotz, M.; Celiksoy, S.; Tuting, L.; Ratzke, C.; Prasad, J.; Ricken, J.; Wegner, S. V.; Ahijado-Guzman, R.; Hugel, T.; Sonnichsen, C. Conformational Dynamics of a Single Protein Monitored for $24 \mathrm{~h}$ at Video Rate. Nano Lett. 2018, 18, 6633-6637.

(24) Tong, L. M.; Wei, H.; Zhang, S. P.; Li, Z. P.; Xu, H. X. Optical properties of single coupled plasmonic nanoparticles. Phys. Chem. Chem. Phys. 2013, 15, 4100-4109.

(25) Visser, E. W. A.; Horaacek, M.; Zijlistra, P. Plasmon Rulers as a Probe for Real-Time Microsecond Conformational Dynamics of Single Molecules. Nano Lett. 2018, 18, 7927-7934.

(26) Chen, H.; Ming, T.; Zhang, S.; Jin, Z.; Yang, B.; Wang, J. Effect of the Dielectric Properties of Substrates on the Scattering Patterns of Gold Nanorods. ACS Nano 2011, 5, 4865-4877.

(27) Hutter, T.; Huang, F. M.; Elliott, S. R.; Mahajan, S. Near-Field Plasmonics of an Individual Dielectric Nanoparticle above a Metallic Substrate. J. Phys. Chem. C 2013, 117, 7784-7790.

(28) Kleemann, M.-E.; Mertens, J.; Zheng, X.; Cormier, S.; Turek, V.; Benz, F.; Chikkaraddy, R.; Deacon, W.; Lombardi, A.; Moshchalkov, V. V.; Vandenbosch, G. A. E.; Baumberg, J. J. Revealing Nanostructures through Plasmon Polarimetry. ACS Nano 2017, 11, $850-855$.

(29) Moreau, A.; Ciraci, C.; Mock, J. J.; Hill, R. T.; Wang, Q.; Wiley, B. J.; Chilkoti, A.; Smith, D. R. Controlled-reflectance surfaces with film-coupled colloidal nanoantennas. Nature 2012, 492, 86-89.

(30) Readman, C.; de Nijs, B.; Szabo, I.; Demetriadou, A.; Greenhalgh, R.; Durkan, C.; Rosta, E.; Scherman, O. A.; Baumberg, J. J. Anomalously Large Spectral Shifts near the Quantum Tunnelling Limit in Plasmonic Rulers with Subatomic Resolution. Nano Lett. 2019, 19, 2051-2058.

(31) Ding, T.; Sigle, D.; Zhang, L.; Mertens, J.; de Nijs, B.; Baumberg, J. Controllable Tuning Plasmonic Coupling with Nanoscale Oxidation. ACS Nano 2015, 9, 6110-6118.

(32) Hill, R. T.; Mock, J. J.; Hucknall, A.; Wolter, S. D.; Jokerst, N. M.; Smith, D. R.; Chilkoti, A. Plasmon Ruler with Angstrom Length Resolution. ACS Nano 2012, 6, 9237-9246.
(33) Mock, J. J.; Hill, R. T.; Tsai, Y.-J.; Chilkoti, A.; Smith, D. R. Probing Dynamically Tunable Localized Surface Plasmon Resonances of Film-Coupled Nanoparticles by Evanescent Wave Excitation. Nano Lett. 2012, 12, 1757-1764.

(34) Hill, R. T.; Mock, J. J.; Urzhumov, Y.; Sebba, D. S.; Oldenburg, S. J.; Chen, S.-Y.; Lazarides, A. A.; Chilkoti, A.; Smith, D. R Leveraging Nanoscale Plasmonic Modes to Achieve Reproducible Enhancement of Light. Nano Lett. 2010, 10, 4150-4154.

(35) Mock, J. J.; Hill, R. T.; Degiron, A.; Zauscher, S.; Chilkoti, A.; Smith, D. R. Distance-dependent plasmon resonant coupling between a gold nanoparticle and gold film. Nano Lett. 2008, 8, 2245-2252.

(36) Hill, R. T.; Kozek, K. M.; Hucknall, A.; Smith, D. R.; Chilkoti, A. Nanoparticle-Film Plasmon Ruler Interrogated with Transmission Visible Spectroscopy. ACS Photonics 2014, 1, 974-984.

(37) Yeshchenko, O. A.; Kozachenko, V. V.; Naumenko, A. P.; Berezovska, N. I.; Kutsevol, N. V.; Chumachenko, V. A.; Haftel, M.; Pinchuk, A. O. Gold nanoparticle plasmon resonance in near-field coupled Au NPs layer/Al film nanostructure: Dependence on metal film thickness. Photonics Nanostruct. Fundam. Appl. 2018, 29, 1-7.

(38) Chou, A.; Vernon, K. C.; Piro, L.; Radi, B.; Jaatinen, E. A.; Davis, T. J. Predicting the Localized Surface Plasmon Resonances of Spherical Nanoparticles on a Substrate: Electrostatic Eigenmode Method. J. Phys. Chem. C 2012, 116, 26517-26522.

(39) Huh, J. H.; Lee, J.; Lee, S. Comparative Study of Plasmonic Resonances between the Roundest and Randomly Faceted $\mathrm{Au}$ Nanoparticles-on-Mirror Cavities. ACS Photonics 2018, 5, 413-421.

(40) de Nijs, B.; Bowman, R. W.; Herrmann, L. O.; Benz, F.; Barrow, S. J.; Mertens, J.; Sigle, D. O.; Chikkaraddy, R.; Eiden, A.; Ferrari, A.; Scherman, O. A.; Baumberg, J. J. Unfolding the contents of sub-nm plasmonic gaps using normalising plasmon resonance spectroscopy. Faraday Discuss. 2015, 178, 185-193.

(41) Hohenester, U.; Truegler, A. MNPBEM - A Matlab toolbox for the simulation of plasmonic nanoparticles. Comput. Phys. Commun. 2012, 183, 370-381.

(42) Waxenegger, J.; Truegler, A.; Hohenester, U. Plasmonics simulations with the MNPBEM toolbox: Consideration of substrates and layer structures. Comput. Phys. Commun. 2015, 193, 138-150.

(43) Kleemann, M. E.; Chikkaraddy, R.; Alexeev, E. M.; Kos, D.; Carnegie, C.; Deacon, W.; de Pury, A. C.; Grosse, C.; de Nijs, B.; Mertens, J.; Tartakovskii, A. I.; Baumberg, J. J. Strong-coupling of WSe2 in ultra-compact plasmonic nanocavities at room temperature. Nat. Commun. 2017, 8, No. 1296.

(44) Yoon, J. H.; Selbach, F.; Schumacher, L.; Jose, J.; Schluecker, S. Surface Plasmon Coupling in Dimers of Gold Nanoparticles: Experiment and Theory for Ideal (Spherical) and Nonideal (Faceted) Building Blocks. ACS Photonics 2019, 6, 642-648.

(45) Lumdee, C.; Yun, B.; Kik, P. G. Effect of surface roughness on substrate-tuned gold nanoparticle gap plasmon resonances. Nanoscale 2015, 7, 4250-4255.

(46) Guzmán, E.; Ritacco, H.; Rubio, J. E. F.; Rubio, R. G.; Ortega, F. Salt-induced changes in the growth of polyelectrolyte layers of poly(diallyl-dimethylammonium chloride) and poly(4-styrene sulfonate of sodium). Soft Matter 2009, 5, 2130-2142.

(47) Webb, H. K.; Vi Khanh, T.; Hasan, J.; Fluke, C.; Crawford, R. J.; Ivanova, E. P. Roughness Parameters for Standard Description of Surface Nanoarchitecture. Scanning 2012, 34, 257-263.

(48) Cirac, C.; Chen, X.; Mock, J. J.; McGuire, F.; Liu, X.; Oh, S.-H.; Smith, D. R. Film-coupled nanoparticles by atomic layer deposition: Comparison with organic spacing layers. Appl. Phys. Lett. 2014, 104, No. 023109.

(49) Sobhani, A.; Manjavacas, A.; Cao, Y.; McClain, M. J.; de Abajo, F. J.; Nordlander, P.; Halas, N. J. Pronounced Linewidth Narrowing of an Aluminum Nanoparticle Plasmon Resonance by Interaction with an Aluminum Metallic Film. Nano Lett. 2015, 15, 6946-6951.

(50) Lei, D. Y.; Fernandez-Dominguez, A. I.; Sonnefraud, Y.; Appavoo, K.; Haglund, R. F.; Pendry, J. B.; Maier, S. A. Revealing Plasmonic Gap Modes in Particle-on-Film Systems Using Dark-Field Spectroscopy. ACS Nano 2012, 6, 1380-1386. 
(51) Stoller, P.; Jacobsen, V.; Sandoghdar, V. Measurement of the complex dielectric constant of a single gold nanoparticle. Opt. Lett. 2006, 31, No. 2474.

(52) Olmon, R. L.; Slovick, B.; Johnson, T. W.; Shelton, D.; Oh, S. H.; Boreman, G. D.; Raschke, M. B. Optical dielectric function of gold. Phys. Rev. B 2012, 86, No. 235147.

(53) Barchiesi, D.; Grosges, T. Fitting the optical constants of gold, silver, chromium, titanium, and aluminum in the visible bandwidth. J. Nanophotonics 2014, 8, No. 083097.

(54) Kongsuwan, N.; Demetriadou, A.; Chikkaraddy, R.; Baumberg, J. J.; Hess, O. Fluorescence enhancement and strong-coupling in faceted plasmonic nanocavities. EPJ Appl. Metamater. 2018, 5, No. 6.

(55) Chikkaraddy, R.; Turek, V. A.; Kongsuwan, N.; Benz, F.; Carnegie, C.; van de Goor, T.; de Nijs, B.; Demetriadou, A.; Hess, O.; Keyser, U. F.; Baumberg, J. J. Mapping Nanoscale Hotspots with Single-Molecule Emitters Assembled into Plasmonic Nanocavities Using DNA Origami. Nano Lett. 2018, 18, 405-411.

(56) Chikkaraddy, R.; de Nijs, B.; Benz, F.; Barrow, S. J.; Scherman, O. A.; Rosta, E.; Demetriadou, A.; Fox, P.; Hess, O.; Baumberg, J. J. Single-molecule strong coupling at room temperature in plasmonic nanocavities. Nature 2016, 535, 127-130.

(57) Kongsuwan, N.; Demetriadou, A.; Chikkaraddy, R.; Benz, F.; Turek, V. A.; Keyser, U. F.; Baumberg, J. J.; Hess, O. Suppressed Quenching and Strong-Coupling of Purcell-Enhanced Single-Molecule Emission in Plasmonic Nanocavities. ACS Photonics 2018, 5, $186-191$.

(58) Hugall, J. T.; Singh, A.; van Hulst, N. F. Plasmonic Cavity Coupling. ACS Photonics 2018, 5, 43-53.

(59) Dionne, J. A.; Baldi, A.; Baum, B.; Ho, C. S.; Jankovic, V.; Naik, G. V.; Narayan, T.; Scholl, J. A.; Zhao, Y. Localized fields, global impact: Industrial applications of resonant plasmonic materials. MRS Bull. 2015, 40, 1138-1145.

(60) Zhan, C.; Chen, X. J.; Yi, J.; Li, J. F.; Wu, D. Y.; Tian, Z. Q. From plasmon-enhanced molecular spectroscopy to plasmonmediated chemical reactions. Nat. Rev. Chem. 2018, 2, 216-230.

(61) Liu, B.; Song, K. R.; Xiao, J. N. Two-Dimensional Optical Metasurfaces: From Plasmons to Dielectrics. Adv. Condens. Matter Phys. 2019, 2019, No. 2329168. 